\title{
TROMBOEMBOLISMO PULMONAR RECURRENTE EN UN PACIENTE CON SÍNDROME ANTIFOSFOLIPÍDICO Y TROMBOCITOPENIA INDUCIDA POR HEPARINA
}

\author{
RECURRENT PULMONARY THROMBOEMBOLISM IN A PATIENT \\ WITH ANTIPHOSPHOLIPID SYNDROME AND THROMBOCYTOPENIA \\ INDUCED BY HEPARIN
}

\section{NAHUEL LITWAK' ${ }^{1}$ MILAGROS SEIJO²}

\begin{abstract}
RESUMEN
El tromboembolismo pulmonar es una urgencia cardiovascular de pronóstico variable y en su estudio etiológico debemos buscar los factores que puedan influir en su génesis y precipitar resultados adversos. La trombocitopenia inducida por heparina y el síndrome antifosfolipídico son enfermedades hematológicas que pueden participar en la fisiopatogenia de tromboembolismo pulmonar y complicar su evolución. A continuación presentamos un caso caso demuestra la ocurrencia de ambas entidades en un paciente.
\end{abstract}

Palabras clave: embolia pulmonar; trombocitopenia; síndrome antifosfolipídico; anticoagulantes.

\section{ABSTRACT}

Pulmonary thromboembolism is a cardiovascular emergency with variable prognosis and in its etiological study we must look for the factors that may influence its genesis and precipitate adverse results.

Heparin-induced thrombocytopenia and the antiphospholipid syndrome are hematological diseases that can participate in the pathophysiology of pulmonary thromboembolism and complicate its evolution. In both cases, they are immune-mediated phenomena that generate a systemic prothrombotic state and require an accurate therapeutic approach.

Keywords: pulmonary embolism; thrombocytopenia; antiphospholipid syndrome; anticoagulants.

REVISTA CONAREC 2018;33(146):251-254 | DOI:10.32407/RCON/2018146/0251-0254

\section{INTRODUCCIÓN}

La trombocitopenia inducida por heparina (HIT) y el síndrome antifosfolipídico (SAF) son enfermedades hematológicas que pueden participar en la fisiopatogenia del tromboembolismo pulmonar (TEP) y agravar su evolución. La coexistencia de estas dos entidades es infrecuente. Se trata en ambos casos de fenómenos inmunomediados que generan un estado sistémico protrombótico. La evidencia relacionada con el abordaje y tratamiento de dichos pacientes es escasa y basada principalmente en reportes de casos y trabajos con series pequeñas de pacientes.

\section{DESARROLLO}

Se presenta el caso de un paciente masculino de 39 años, extabaquista y con antecedente de trombosis venosa profunda (TVP) en el año

\footnotetext{
1 Residente de Cardiología

2 Jefe de Residentes. Hospital Universitario Fundación Favaloro. CABA, Rep. Argentina.
}

$\triangle$ Correspondencia: Dr. Nahuel Litwak. Av. Santa Fe 2534. C1425BGN CABA, Rep. Argentina.nahuel.litwak@gmail.com.

Los autores declaran no tener conflictos de intereses.

Recibido: 10/11/2017| Aceptado: 02/02/2018
2002 por la cual recibió tratamiento anticoagulante con antagonistas de la vitamina K (AVK). Se internó inicialmente en otro centro por encontrarse sintomático para disnea en clase funcional (CF) III asociado a 2 episodios autolimitados de hemoptisis. En dicho centro se realizó diagnóstico de TVP y TEP agudo por lo que recibió tratamiento con heparina sódica. Presentó en el curso de su internación plaquetopenia que alcanzó valores de hasta $37 \mathrm{mil} / \mathrm{mm}^{3}$, interpretándose cuadro como probable HIT, sin disponer de pruebas confirmatorias. Se completó su estudio mediante centellograma ventilación/perfusión que informó ausencia de perfusión pulmonar izquierda y defectos segmentarios de la perfusión pulmonar derecha, con alta probabilidad de TEP. Se realizó cateterismo pulmonar con presión arterial pulmonar media de $47 \mathrm{mmHg}$ y resistencias pulmonares elevadas de 29 unidades Wood. Los análisis de laboratorio con factor antinuclear y serologías virales fueron negativas. Luego de 33 días de internación, se otorgó su egreso hospitalario bajo anticoagulación con acenocumarol y tratamiento de la hipertensión pulmonar con sildenafil y bosentán.

Posterior a su externación, fue evaluado en consultorios externos por persistir con disnea CF III, para descartar hipertensión pulmonar (HTP) tromboembólica crónica. Previo a la realización de los exámenes complementarios solicitados, presentó nuevamente 2 episodios de hemoptisis autolimitados asociados a progresión de su disnea hasta CF IV estando bajo tratamiento anticoagulante. Se realizó angiotomografía computarizada de tórax (Figuras 1 y 2) que informó la presencia de defectos de relleno endovascular en rama segmentaria del ló- 


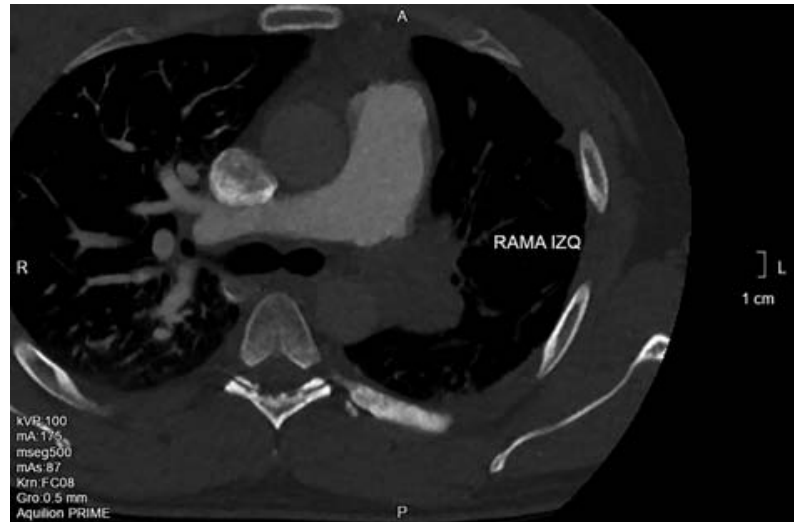

Figura 1. Tomografía computarizada con contraste endovenoso. Se evidencia dilatación del tronco de la arteria pulmonar asociado a defecto de relleno de contraste en la rama izquierda de la misma. Izq: izquierda.

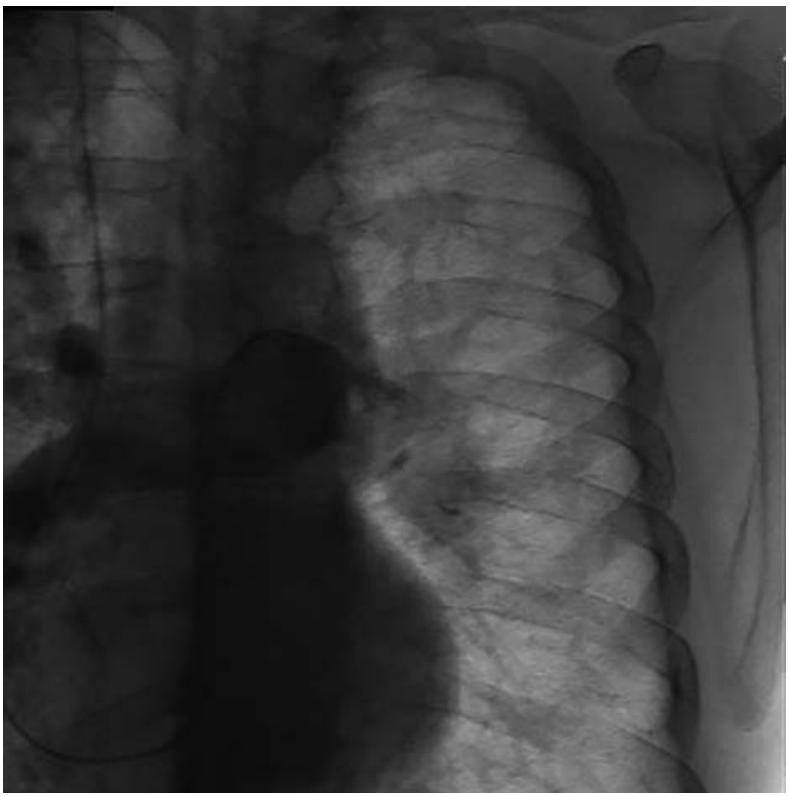

Figura 3. Angiografía pulmonar con oclusión de arteria pulmonar izquierda en origen y oclusión subsegmentaria derecha por completa.

bulo medio e inferior derechos, que permitieron interpretar el cuadro clínico como nuevo episodio de TEP derecho agudo, por lo que se decidió su internación

A su ingreso, se encontraba lúcido, hemodinámicamente estable, normotenso (presión arterial de 130/80 mmHg), frecuencia cardiaca de 85 latidos por minuto, taquipneico, con una saturación por oximetría de pulso de $88 \%$ al aire ambiente que corregía con oxígeno suplementario en bajas dosis. En la analítica del laboratorio presentó plaquetopenia (recuento de plaquetas: $91 \mathrm{mil} / \mathrm{mm}^{3}$ ) con el siguiente coagulograma: tiempo de protombina: 49\%, RIN (Rango internacional normatizado): 1,65; tiempo de tromboplastina parcial activado: 64 segundos. Se consensuó con el servicio de Hematología la anticoagulación con rivaroxaban dada la sospecha previa de HIT y ausencia de disponibilidad de fondaparinux y se procedió a la colocación de filtro de vena cava inferior. Se continuó su evaluación mediante angiografía pulmonar que reveló oclusión de arteria pulmonar izquierda y subsegmentaria de-

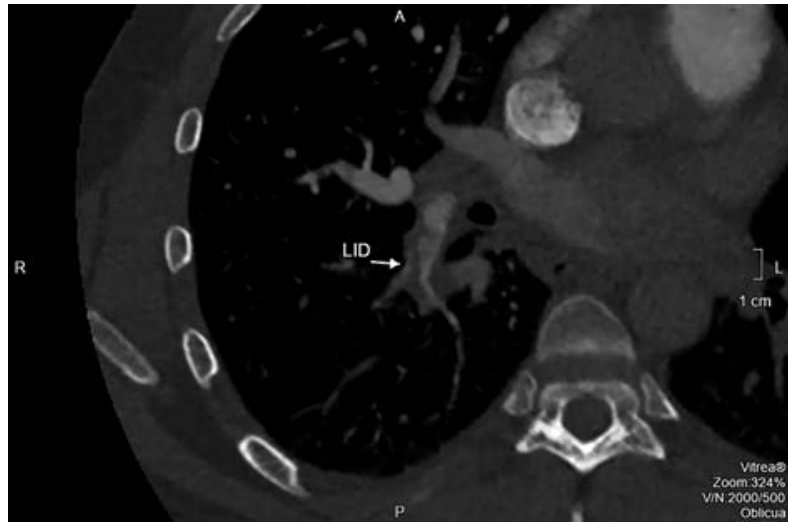

Figura 2. Tomografía computarizada con contraste endovenoso. Se evidencia defecto de relleno de contraste en la rama lobar inferior derecha compatible con tromboembolismo pulmonar agudo. LID: lóbulo inferior derecho

recha con HTP severa (Figura 3), cinecoronariografía con arterias coronarias sin lesiones angiográficamente significativas, con circulación colateral desde la arteria circunfleja hacia la circulación pulmonar. Se realizó un ecocardiograma Doppler que evidenció dilatación severa de las cavidades derechas con deterioro moderado de la función sistólica del ventrículo derecho, presión sistólica pulmonar de 88 mmHg e insuficiencia tricuspídea moderada. Los análisis de laboratorio confirmaron el diagnóstico de SAF con triple positividad de anticuerpos (anticoagulante lúpico, anticuerpos anti-ß2 Glicoproteína I y anti-cardiolipina positivos) y prueba de ELISA con antianticuerpos antifactor plaquetario 4 (anti-PF4) negativos. Luego de 8 días de internación, se otorgó el egreso hospitalario bajo tratamiento anticoagulante con rivaroxaban, con corticoides orales por interpretarse plaquetopenia de origen inmune y seguimiento ambulatorio por los servicios de Cardiología, Neumonología y Hematología.

A los 15 días del egreso, consultó nuevamente al departamento de emergencias por hemoptisis asociado a progresión de su disnea hasta CF IV Se realizó una nueva angiotomografía pulmonar que evidenció imagen compatible con infarto pulmonar hemorrágico (Figura 4). Se suspendió en dicho contexto la anticoagulación con rivaroxaban y luego de 3 días de estabilidad hemodinámica con recuento plaquetario en ascenso, se reinició anticoagulación con warfarina. Se reevaluó la angiografía previamente realizada en conjunto con el servicio de Hemodinamia y se procedió a la embolización con coils de ramas de circulación colateral de arteria mamaria izquierda hacia el hilio pulmonar izquierdo (Figura 5) Evolucionó favorablemente por lo que se decidió su externación.

Un mes más tarde fue internado en otro centro con diagnóstico de TEP agudo donde se indicó tratamiento anticoagulante con heparina sódica evolucionando con plaquetopenia, falleciendo 48 hs después de su ingreso por hemoptisis masiva.

\section{DISCUSIÓN}

La trombocitopenia inducida por heparina (HIT) es una de las complicaciones no hemorrágicas más importantes de la utilización de la heparina y sus derivados en la práctica clínica'. La misma se produce como consecuencia del desarrollo de anticuerpos contra complejos entre heparina y una proteína plaquetaria: el factor plaquetario 4. El desarrollo de estos an- 


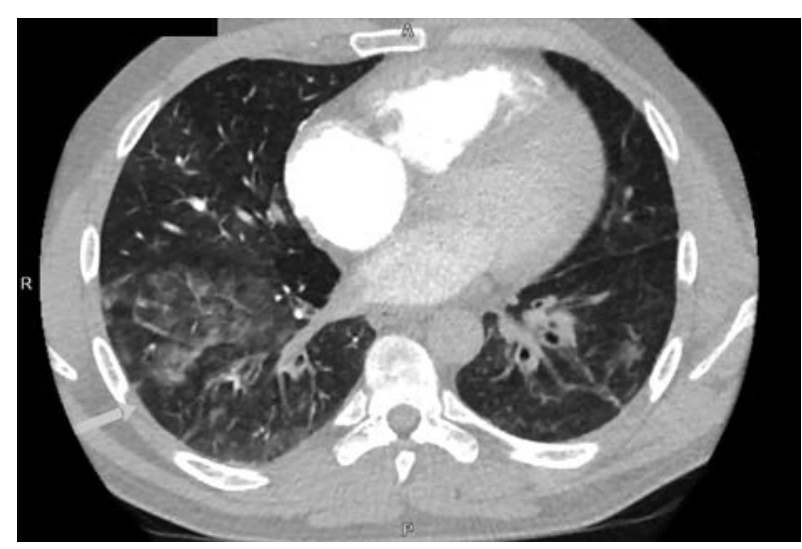

Figura 4. Signos de ocupación alveolar que se manifiestan como opacidades centroacinares en vidrio esmerilado en ambas bases pulmonares a predominio derecho que podrían estar en relación con hemorragia alveolar en primer término.

ticuerpos se produce en el 8 y $17 \%$ de los pacientes según la serie revisada ${ }^{1-4}$, alcanzando proporciones más elevadas en pacientes luego de cirugía cardíaca 5 . Sin embargo, la trombocitopenia y los fenómenos trombóticos venosos o arteriales son más infrecuentes, afectando del 0,1 al 5\% de los pacientes expuestos a la droga. Dichos anticuerpos son capaces de unirse al receptor plaquetario y leucocitario para el fragmento constante de las inmunoglobulinas lgG (FcyRlla), promoviendo la liberación de trombina, factor tisular y generando de esta forma una señalización intracelular que culmina con la activación plaquetaria y liberación de partículas procoagulantes. Uno de los scores más utilizados en la práctica clínica es el "4T's" con alta sensibilidad y alto valor predictivo negativo para aquellos pacientes categorizados con baja probabilidad de presentar la enfermedad ${ }^{6}$; actualmente otros scores se encuentran en revisión? 7 La sugerencia actual consiste en utilizar de forma conjunta dichos parámetros asociados a la demostración serológica de los anticuerpos mediante inmunoensayo o una prueba funcional con ensayos de activación plaquetaria. Para el tratamiento se deberá discontinuar el tratamiento con todo tipo de heparinas y evitar la re-exposición a las mismas, salvo en ciertos casos con seronegativización de los anticuerpos y necesidad de cirugía cardiovascular. Se indicará anticoagulación durante un período de tiempo variable según la condición clínica del paciente, siendo las opciones más evaluadas hasta la actualidad el argatrobán, bivalirudina, danaparoide y fondaparinux. Estos últimos no siempre se encuentran disponibles, surgiendo el interrogante de la utilidad de los anticoagulantes orales directos (DOACs) con experiencia en el tratamiento de múltiples patologías, dadas sus escasas interacciones farmacológicas, su administración en dosis fijas y la ausencia de necesidad de monitoreo rutinario. La evidencia actual para el HIT es escasa, surgiendo fundamentalmente de reportes de casos, siendo necesarios estudios prospectivos y multicéntricos que determinen su eficacia y seguridad ${ }^{8,9}$.

\section{BIBLIOGRAFÍA}

1. Arepally GM. Heparin-induced Thrombocytopenia. Blood 2017;129(21):28642872.

2. Warkentin TE, Levine MN, Hirsh J, Horsewood P, Roberts RS, Tech M, et Al. Hepa-

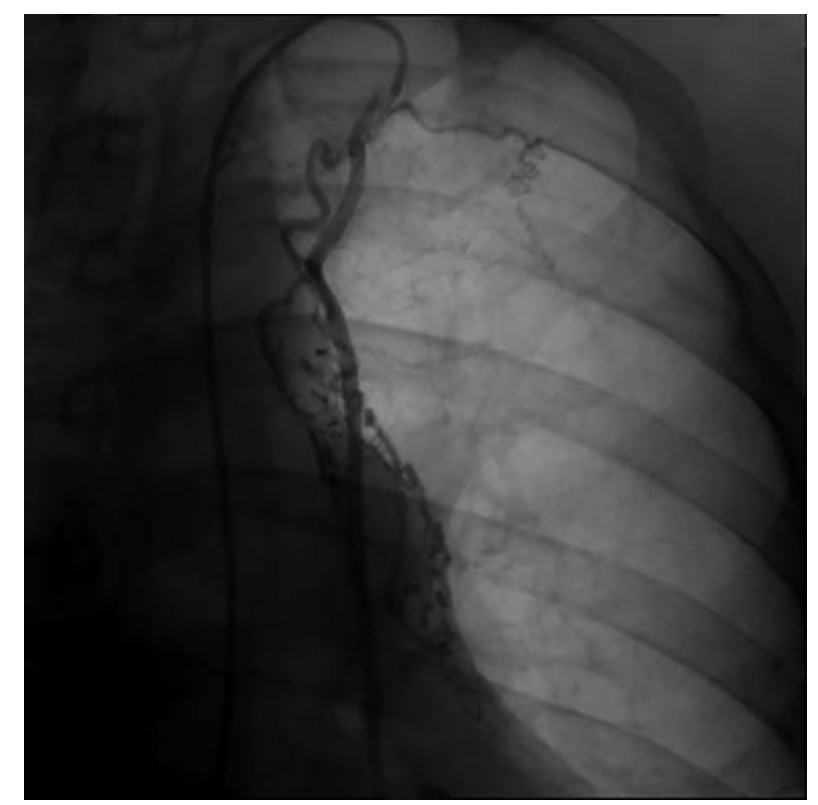

Figura 5. Angiografía de la arteria mamaria interna izquierda con circulación colateral hacia la arteria pulmonar izquierda.

EI SAF es una enfermedad sistémica adquirida que requiere para su diagnóstico la coexistencia de criterios clínicos, basados en fenómenos trombóticos, arteriales o de comorbilidades obstétricas, asociados criterios bioquímicos con positividad para uno o más anticuerpos antifosfolipídicos de forma sostenida en el tiempo ${ }^{10}$. Dada la elevada frecuencia de fenómenos trombóticos e incluso recurrencia de los mismos, el estándar de tratamiento consiste en su anticoagulación con AVK, siendo escasa la evidencia para la utilización de los DOACs ${ }^{11}$

La coexistencia de ambas enfermedades resulta extremadamente infrecuente $^{12,13}$, existiendo en la literatura sólo algunos reportes de casos.

En el paciente presentado, luego de la sospecha de HIT, se suspendió el tratamiento con heparinas, y dada la falta de disponibilidad de otras opciones terapéuticas como fondaparinux y argatrobán ${ }^{14}$, se debió iniciar anticoagulación con DOACs a pesar de la limitada evidencia en su utilización.

\section{CONCLUSIÓN}

La coexistencia del síndrome antifosfolipídico y trombocitopenia inducida por heparina es infrecuente. Este caso demuestra la ocurrencia de ambas entidades en un paciente. La evidencia relacionada con el abordaje y tratamiento de dichos pacientes es escasa y basada principalmente en reportes de casos y trabajos con series pequeñas de pacientes. Asimismo, refleja el difícil manejo de estos pacientes que obliga al abordaje multidisciplinario de la patología. 
duced Thrombocytopenia in a large, tertiary care teaching hospital. Chest 2007;131(6):1644-1649.

4. Salter BS, Weiner MM, Trinh MA, Heller J, Evans AS, Adams DH, et al. Heparin-Induced Thrombocytopenia: A Comprehensive Clinical Review. I Am Coll Cardiol 2016;67(21):2519-2532.

5. Trossaert M, Gaillard A, Commin PL, Amiral J, Vissac AM, Fressinaud E. High incidence of anti-heparin/platelet factor 4 antibodies after cardiopulmonary bypass surgery. Br J Haematol 1998;101(4):653-5.

6. Lo GK, Juhl D, Warkentin TE, Sigouin CS, Eichler P, Greinacher A. Evaluation of pretest clinical score (4 T's) for the diagnosis of heparin-induced thrombocytopenia in two clinical settings. J Thromb Haemost 2006;4(4):759-65.

7. Joseph L, Gomes MPV, Solaiman FA, St John J, Ozaki A, Raju M, et al. External Validation Of The HIT Expert Probability (HEP) Score, a Novel Pre-Test Probability Model For Heparin-Induced Thrombocytopenia Based On Broad Expert Opinion. Blood 2013;122(21):1699.

8. Seculini Patiño CE, Tabares AH. Podrían ser los anticoagulantes orales directos (ACODs) una alternativa para el tratamiento de la trombocitopenia inducida por heparina? Rev Fed Arg Cardiol 2016;45(1):8-12.
9. Warkentin TE, Pai M, Linkins LA. Direct oral anticoagulants for treatment of HIT: update of Hamilton experience and literature review. Blood 2017;130(9):11041113.

10. Chaturvedi S, McCrae KR. Diagnosis and management of the antiphospholipid syndrome. Blood Rev 2017;31(6):406-417.

11. Dufrost V, Risse J, Zuily S, Wahl D. Direct Oral Anticoagulants Use in Antiphospholipid Syndrome: Are These Drugs an Effective and Safe Alternative to Warfarin? A Systematic Review of the Literature. Curr Rheumatol Rep 2016;18(12):74.

12. Adediran S, Agostino N. Coexistence of Antiphospholipid Syndrome and Heparin-Induced Thrombocytopenia in a Patient with Recurrent Venous Thromboem bolism. Case Rep in Hematol 2017;2017:3423548.

13. Abd-Elsayed AA, Lisco SJ, Alsidawi S, Bonomo J. Simultaneous antiphospholipid syndrome and heparin-induced thrombocytopenia in a single patient. A A Case Rep 2014;2(1):9-10.

14. de Jesus GR, Agmon-Levin N, Andrade CA, Andreoli L, Chighizola CB, Porter TF, et Al. 14th International Congress on Antiphospholipid Antibodies Task Force report on obstetric antiphospholipid syndrome. Autoimmun Rev 2014;13(8):795-813. 